\title{
APPLYING A PERFORMANCE-FOCUSED RACE-YACHT METHODOLOGY TO THE DESIGN OF A SUPERYACHT
}

\author{
R G Shaw, Unitec Institute of Technology, New Zealand
}

\section{SUMMARY}

A background in designing and sailing custom high-performance race yachts unrestricted by rating rules and formulas has fostered a penchant to prioritise performance and enjoyment of sailing in each new design. This project explores the possibilities for performance, function and form achievable through refocusing the design drivers in the development of a superyacht, with a fresh emphasis on the design and engineering aspects of construction, hull form, sail plan and appendages. This design prioritises performance over the traditional aesthetics, features and accommodations often associated with superyachts, while maintaining superyacht stature and appeal. Drawing on knowledge gained from the development of smaller coastal and offshore performance race-yachts, this project explores how to capitalise on many of the technologies that have been refined in these smaller yachts. This refocus of concept has resulted in a departure from the traditional superyacht style, offering high performance coupled with unique and tasteful aesthetics, appealing to those seeking a more visceral sailing experience from a superyacht.

\section{NOMENCLATURE}

CFD computational fluid dynamics

IRC Royal Ocean Racing Club international measurement and rating rule

MCA Maritime and Coastguard Agency

VPP velocity prediction program

\section{INTRODUCTION}

Experience developing a series of prototype yacht designs prioritising performance through a focus on innovation and refinement has seen development driven through a unique lens. With innovation unrestricted by rating rules in the quest for unmatched performance, the principal parameters are set by size, crew and safety considerations. Shaw Yacht Design has developed several successful new designs, each time refining this approach to achieve unique performance-focused yachts that are pure of purpose.

With the design of performance-based racing yachts, the principal design drivers are centred on accessibility (in terms of cost and ease of construction, and transportability of the finished boat, performance (including quantifiable elements such as speed) and handling characteristics/feel (user experience). An iterative design process examining each aspect is applied to achieve maximum potential when considered against the principal design criteria. The design process is a combination of art, utilising the designer's eye, innate knowledge and experience, and science, with recourse to computer-aided technologies in the fields of engineering and aero-hydrodynamics.

However, designing race yachts restricted only by budget (setting the size parameters) and transportability, without the constraints of rating rules and charter requirements (e.g. IRC, MCA), has led to a freedom of design methodology which cannot be applied wholeheartedly to designs which bring different challenges related to engineering and sailing systems.

With this in mind, this paper will explore the application of a race-yacht design methodology to the design of a 50 metre superyacht, which would offer not only aspects of superyacht-quality accommodation and comfort of experience but also race-yacht type performance. This kind of yacht would appeal to owners who wish for greater performance and tactility from their superyacht and who wish to take part in racing events and regattas around the world, such as New Zealand's Millennium Cup, without sacrificing the comfort and luxury to which they are accustomed.

The result is a unique, purposeful yet elegant 50 metre performance-focused superyacht. It combines the performance of a pure race boat with the safety and handling features required of a superyacht.

\section{DESIGN METHODOLOGY}

The process of yacht design is complex and draws on a range of knowledge and expertise. The blending of art and science is apparent within the design endeavour but, along with the amalgamation of theory and practice, is not always articulated well by yacht designers. Larsson and Eliasson concede that "Yacht design is an iterative, "trial and error' procedure” involving a design spiral, "where the designer runs through all the design steps and then returns to the starting point, whereupon a new 'turn' begins." [1]

The iterative nature of the design process lends itself to action research, as this method accommodates the cyclical process of identifying problems, analysing them and 
taking action to address issues before evaluating the results and moving into another cycle (Swann, 2002). Each iteration of the process adds to the theory.

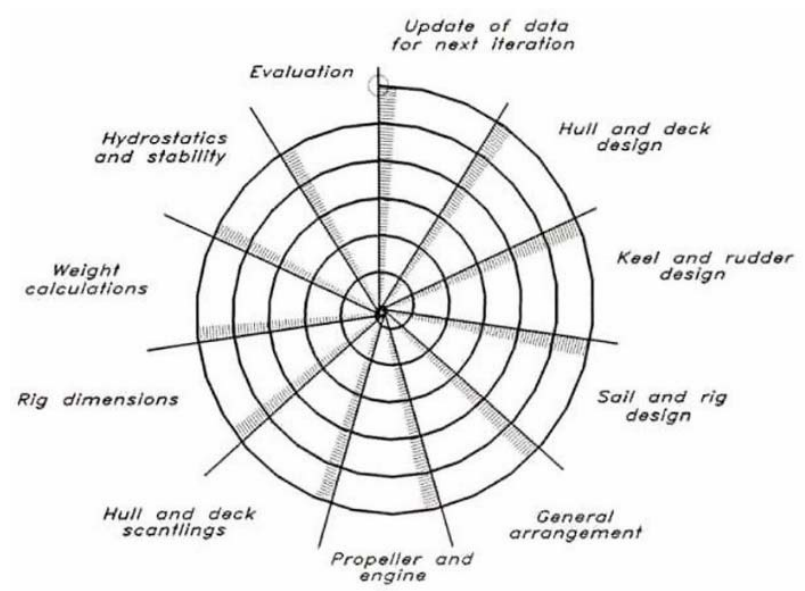

Figure 1: The yacht design spiral (Larsson and Eliasson, 1994, p. 5).

Along with the various definitions and explanations of action research there are also many diagrammatical interpretations of the process. For the purposes of this design the elements of planning, acting, observing and reflecting illustrated by Zuber-Skerritt [2] have been used to explain the project. This illustration of the action research cycles interrelates well with the notion of yacht design as a complex, spiral process (as articulated by Larsson \& Eliasson, see above).
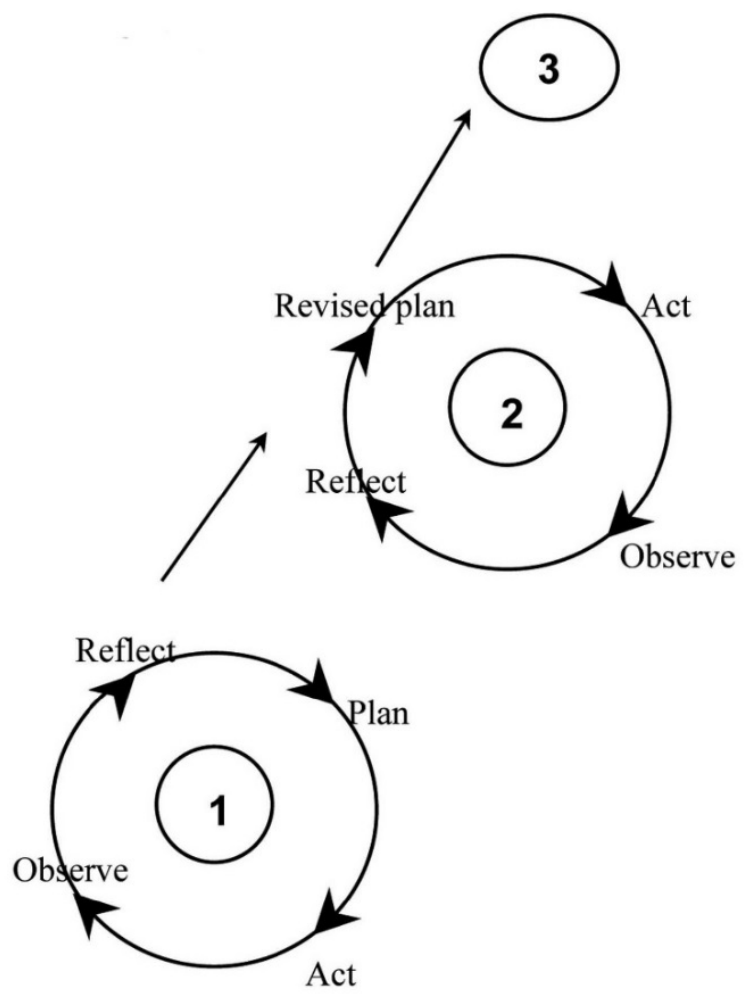

Figure 2: The action research spiral (Zuber-Skerritt, 2001, p.20).
Applying this action research method to the development of a performance race yacht, the Planning phase for each aspect of the design includes identifying current challenges and the foundations of them, including conventional wisdom and limitations on construction and knowledge. The Acting phase is evident in relation to each of the design aspects, as a process is put in place to respond to the identified challenge. Observation is apparent in the information gathered during the development for each of the aspects - a key step, as the systematic gathering of information is what distinguishes this journey from alternative processes of designing a yacht and contributes research knowledge to the field. Reflection on the action is then used to inform the next iteration of the process.

A critical component of this design method is the firsthand knowledge and feedback gained through extensive sailing on a range of yachts and primarily on yachts designed through this process. Extensive sailing on these designs, often in a competitive environment, also exposes these designs to critique and unrestricted feedback in the public domain. This extensive verification and analysis forms a critical component of informing the design of future models. As this design method puts a higher value on the designer's intuition and personal judgement, it critical that these personal skills be well informed, current and constantly updated.

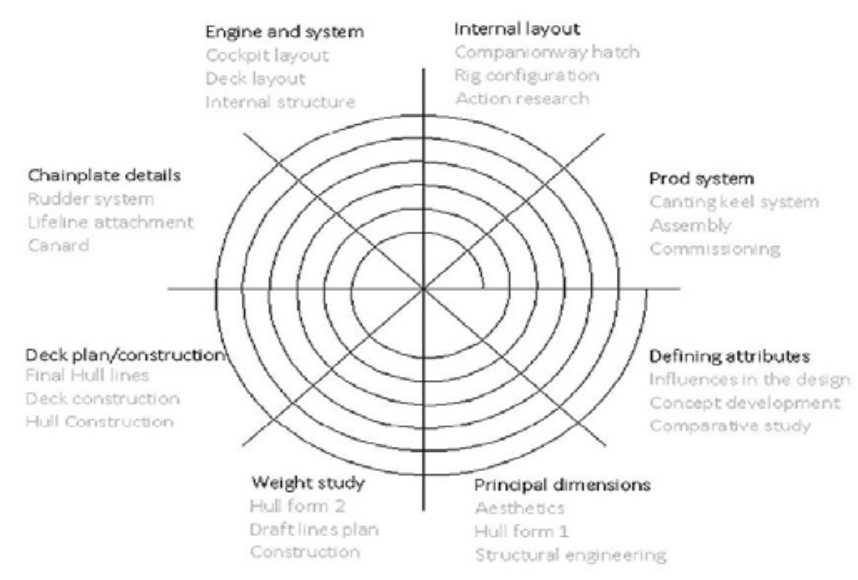

Figure 3: Example of a design spiral previously utilised by Shaw Yacht Design.

This designer-led approach to development and refinement is in contrast to the alternative of computational analysis, which does not provide precise feedback on how design variations will manifest in the yacht's complete sailing performance package. The designer-led approach accepts that the designer must apply his own judgement to achieve a well-balanced, allround-performing yacht through analysis of the yacht's variables and complexities. These limitations of computer modelling are noted by several authors, including Levadou, Prins, \& Raven [3], and Roux et al. [4], who state, 
One of the major difficulties of such a computation [using CFD programs] is that the flow over any one of the components — sails and hull - operating in a real sailing boat is a very complex combination of many phenomena, some of which being clearly non-linear. Besides this, a sailing boat is an integrated system in which sails and hull closely interact.

Larsson also notes that "A weak point of most [computer] VPPs is the prediction of the performance in waves ... Waves create effects in all degrees of freedom . . . therefore a complete model for the wave effects is out of reach at present." [5]

\section{ADAPTATION OF THE DESIGN METHODOLOGY TO SUPERYACHT DESIGN}

This design method, previously applied to performance race yachts, can with some adaptation be applied to the development of a much larger superyacht. While the pure design elements remain unchanged and assist the creative process, some adaption is necessary in specific areas. Safety is always a major design consideration in any yacht, large or small. However, as yachts increase in size more emphasis needs to be placed on the safety of the crew due to the increased loads generated. For example, if a mainsail fails under load on a small boat and the unsupported boom fell to the deck, the risk of injury to a crew member or serious damage to the boat is minimal. If this scenario was repeated on a 50 metre yacht the risk to the crew and the boat is very high and could result in death. The consideration of the dangers of dealing with such high loads must be followed through in all aspects of the superyacht design including structural considerations, sails, sheets, hardware, rig design and hydraulics.

Another area that requires different treatment is related to the yacht's draft. In the case of the small yacht, draft is a relatively minor consideration and is most often limited by identifying a practical balance between righting moment, hydrodynamic considerations and any local draft restrictions. However, in a superyacht design draft becomes a major consideration as considerable draft is required to provide adequate righting moment to balance the increased sail plan, yet this can easily become unwieldy and impractical in terms of transporting the boat and use of marina and port facilities. Therefore the use of both a canting and lifting keel system must be explored, to maximise righting moment without excessive draft and enable entry to reduced-draft facilities.

Developing the overall concept design through refining each specific design aspect involves engaging with the action research model described above: planning a design action, acting on that, observing the effects of that plan on the design, and reflecting on how each aspect could be altered and improved in a further iterative spiral.

This type of design development process lends itself well to the practice of iterative design and engineering. This can be a great benefit to the development of a superyacht project by potentially speeding up the delivery time from project conception through design and engineering development, to construction and delivery.

\section{DESIGN ASPECTS}

The overall aim of the project is to consider the development of a performance superyacht through an alternate lens previously applied to the development of performance race yachts. This approach of shifting the emphasis to focus on performance and pure sailing experience will produce a unique yacht which although complex of design provides a more tactile and rewarding, user-focused sailing experience.

\subsection{DESIGN DRIVERS}

The key design considerations are:

- Usability (reliability, safety)

- $\quad$ Performance (including quantifiable elements such as speed)

- Handling characteristics/feel (user experience).

The design development follows an iterative design development process, from the initial concept design through to final design and construction, covering the consideration of aesthetics, hull and deck form, appendages, and rig and sail dynamics. Each of these are assessed in terms of their impact on the key considerations of usability, performance, and handling characteristics.

The objective to bring creativity to the fore in the design and development process, complemented by specific engineering expertise, brings a shift in the development focus from the science-based, tangible drivers such as compliance to regulations and physical characteristics to less tangible concepts such as user experience and feel (see figure 4). Framing the yacht design problem and objectives in this context changes the order of priority by putting the human experience first and requiring the engineering requirements to follow and adapt to or inform these, rather than drive them.

\section{TANGIBLE}

Project objectives

Development method

Componentry

Hydrostatics

Physical characteristics

\section{INTANGIBLE}

Deceleration into waves

User experience

Performance

Fluid dynamics

Feel
Figure 4: Tangible and intangible aspects of the design process. This chart shows how aspects of both art and science are utilised in the process. 


\section{1 (a) Usability}

A key and defining design objective with this type of yacht is Usability. To develop a superyacht with a serious performance focus requires a shift in philosophy for this type of yacht away from the traditional high specification of potentially every aspect of the yacht to a focus on the primary performance-related elements of the yacht. By cutting away the other elements we so often associate with a yacht of this type it is possible to create a large superyacht that is a real joy to sail for skipper and crew.

By placing a focus on usability, attention must also be paid to safety considerations, especially in the management of high loads when in sailing configuration. The yacht also needs to be reliable in its performance and operation.

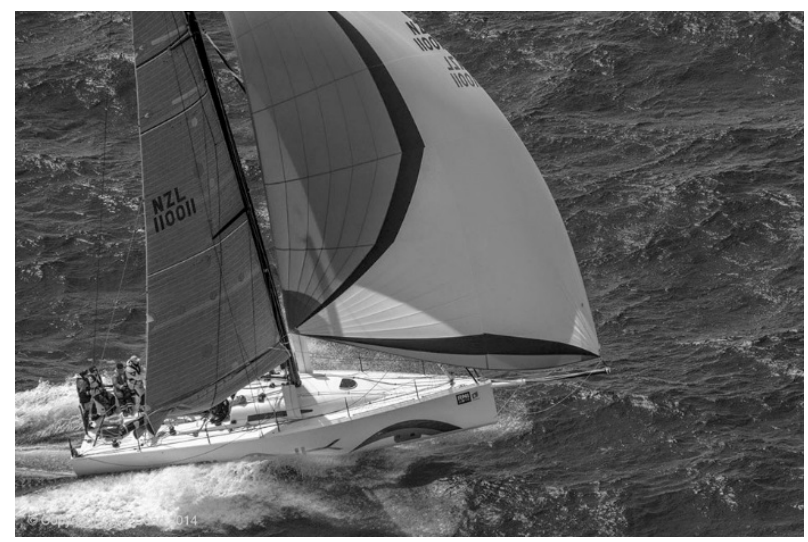

Figure 5. Shaw 12 metre cruiser-racer Blink sets a record across the Cook Strait. (Photo: Chris Coad)

\section{1 (b) Performance}

The second important objective is the yacht's performance, the overall aim being to produce a very high performance yacht in the 50 metre size range, with good all-round abilities in a wide range of conditions, both wind strengths and true wind angles. This would be measured in terms of observed performance and race results once the boat is launched. However, Skene states:

The problem of designing a sailing yacht with speed as a foremost consideration is a most complex one. External conditions to which a yacht is subject, such as force and direction of wind, condition of sea, etc., are constantly changing so that the attainment of a given speed may not be sought, but rather such a form as shall be easily driven at all speeds within appropriate limits ... A harmonious adjustment between power and resistance should be sought. [6]

\section{1. (c) Handling characteristics/“feel”}

The third aspect is less tangible: the yacht's handling characteristics and the experience it provides the user in terms of its "feel" and ease of use when being sailed. This cannot be quantified and scientifically described but can be observed once that yacht has been launched. This aspect is therefore informed by science and engineering but in terms of analysis falls more in the realm of "art".

A yacht cannot merely be fast in terms of straight-line speed; if it is difficult for the crew to extract this performance or the boat is uncomfortable and inconvenient to sail well then the design cannot be considered successful or to have fulfilled its purpose.

\subsection{DESIGN DETAIL}

The defining features of the 50 metre concept superyacht developed through the application of this design method would evolve as the project progressed and with the inputs, requirements and biases of the client, designers and engineers. However, conceptually it is expected to be defined by the following attributes:

- The keel would likely be both canting and retracting, to accommodate the considerable draft to achieve a high righting moment without excessive ballast while also being able to provide reasonable access to ports and desirable anchorages. This would provide some interesting engineering challenges but is not unachievable. This keel configuration, including the need for a lifting foil or foils, would also have a significant impact on the interior accommodation of the yacht but this is consistent with the concept and focus of the design.

- A twin-rudder configuration would complete the appendage package. This would be a good fit with the beam and hull form consistent with a powerful yet light displacement hull of this type of yacht. Having twin rudders would also enable the rudder draft to be short enough not to require the rudders to be retracted as the boat entered shallow ports which would require the keel to be lifted.

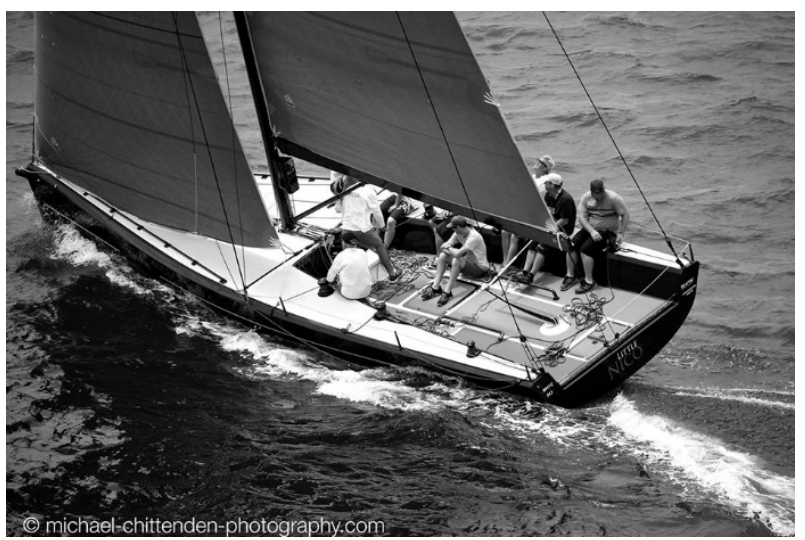

Figure 5. Shaw 11 metre Little Nico sheets on upwind for the first time on its maiden voyage. (Photo: Michael Chittenden)

- $\quad$ The yacht would potentially have a flush deck with a large cockpit space, but with the potential for a low- 
profile deckhouse or transparent deck to allow light and visual connection from the yacht's interior to exterior.

- $\quad$ As the design is not rule bound, it is open to exploit full use of heavily automated sail-handling systems. Winches can be hydraulically driven, enabling the boat to be sailed by a significantly reduced number of crew to a traditional race boat of this size and performance capability.

- $\quad$ Advancement in rigs and rigging technology and sail construction make it feasible to have a yacht of this size sail efficiently with a single mast sloop rig configuration. This is also made possible through automation of the sail handling systems. Utilising current advancements in this area, sails of that size can be safely controlled without needing huge numbers of crew. This is enables a big gain in performance, as the sails can be trimmed actively far more effectively than would be possible with a yacht that relied solely on people power to trim the sails. This provides a gain both in terms of weight saving but also sail-trim efficiency.

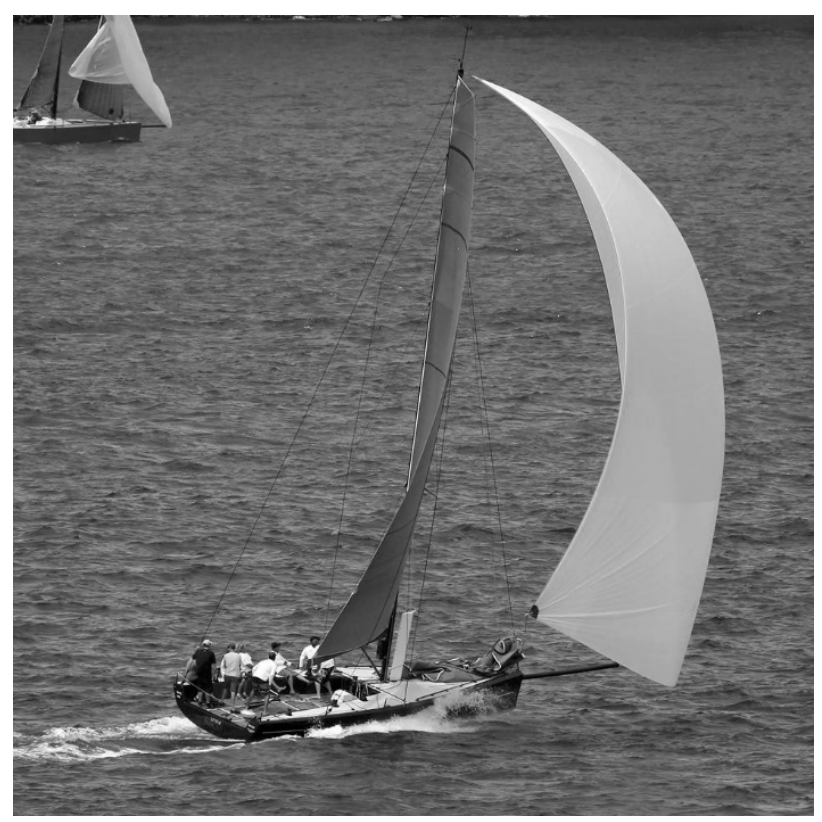

Figure 6. Shaw 11 metre Little Nico powers away downwind on Sydney Harbour. (Photo: Michael Chittenden)

The focus of this design is to achieve an elegant but efficient and high-performance yacht with emphasis on sailing performance. Although not a cruising boat in the style of a resort or luxury hotel, it has potential to offer quality short-term accommodation for owners and guests and crew accommodation. This accommodation would be of a minimal level when compared with most current superyachts of a similar size but is adequate and consistent with the purpose of this yacht.
It is envisaged that support facilities and recreational equipment would be accommodated on a separate tender to ensure the yacht itself wouldn't be compromised by systems and equipment that would detract from the yacht's primary objectives.

Due to the inherent performance, delivery between destinations and events would primarily be under sail rather than relying on a transporter.

In summary, some defining attributes of this yacht that would set it apart from her contemporaries are:

- Full carbon construction.

- Significantly lower displacement-length ratio

- Higher power-to-weight ratio.

- Deeper draft and lifting, canting keel.

- Maximum use of automated sail-handling systems.

- $\quad$ Reduced interior accommodations.

These features will increase performance and make the boat more enjoyable to sail and more responsive.

\section{CONCLUSION}

The development of a 50 metre performance-oriented superyacht provides a platform for the exploration of the inter-relationship of art and science in the practice of yacht design. The design journey underscores the need to observe and reflect, to consider conventional wisdom and explore ways of expanding it, drawing on a balance of science (mathematical- and computer-driven processes) and art (intuition, past experience and the “designer's eye”).

The action research method and use of a design spiral methodology used to develop smaller race yachts can be easily adapted to this larger-scale project. Focusing on the key considerations mentioned above, each aspect of the design, including the overall look (aesthetics), construction methods, hull and deck design, keel and appendages, and rig and sail design, can be examined and developed using the process of planning, acting, observing and reflecting, leading on to the next iteration of each aspect and its development.

The resulting design will be a well-performing, fast and powerful yacht which is enjoyable to sail and aesthetically pleasing, offering a high-quality user experience for both owner and crew. Such a yacht should be a pleasure to sail, responsive and with good handling. The design process will direct the focus away from the user experience when the boat is at anchor, and redirect it to maximising the experience when it is under sail.

\section{REFERENCES}

1. L. Larsson \& R.E. Eliasson, Principles of yacht design. London, UK: Adlard Coles, 1994, p. 5. 
2. O. Zuber-Skerritt, 'Action learning and action research: paradigm, praxis and programs.’ In S. Samkaram, B. Dick, R. Passfield, \& P. Swepson (eds). Effective Change Management Using Action Research and Action Learning: Concepts, frameworks, processes and applications. Southern Cross University Press: Lismore, Australia, 2001, pp. 1-20.

3. M.M.D. Levadou, H.J. Prins \& H.C. Raven, 'Application of advanced computational fluid dynamics in yacht design.' Paper presented at the 15th International HISWA Symposium on Yacht Design and Yacht Construction, Amsterdam, 1998.

4. Y. Roux, S. Huberson, F. Hauville, J. Boin, M. Guilbaud, \& M. Ba, 'Yacht performance prediction: towards a numerical VPP.' Paper presented to the High
Performance Yacht Design Conference, Auckland, 4-6 December 2002.

5. L. Larsson, L. 'Scientific methods in yacht design.' Annual Review of Fluid Mechanics, 22, 1990, pp. 32985.

6. N.L. Skene, Elements of Yacht Design. New York, NY: Kennedy Bros, 1937, pp.10-11.

\section{AUTHOR BIOGRAPHY}

Robert Shaw is a lecturer at Unitec Institute of Technology in Auckland, New Zealand, and the principal of performance yacht design studio Shaw Yacht Design. 